\title{
Adaptive Feedback Synchronization of a General Complex Dynamical Network With Delayed Nodes
}

\author{
Qunjiao Zhang, Junan Lu, Jinhu Lü, Senior Member, IEEE, and Chi K. Tse, Fellow, IEEE
}

\begin{abstract}
In the past decade, complex networks have attracted much attention from various fields of sciences and engineering. Synchronization is a typical collective behavior of complex networks that has been extensively investigated in recent years. To reveal the dynamical mechanism of synchronization in complex networks with time delays, a general complex dynamical network with delayed nodes is further studied. Based on a suitable model, we investigate the adaptive feedback synchronization and obtain several novel criteria for globally exponentially asymptotic synchronization. In particular, our hypotheses and the proposed adaptive controllers for network synchronization are very simple and can be readily applied in practical applications. Finally, numerical simulations are provided to illustrate the effectiveness of the proposed synchronization criteria.
\end{abstract}

Index Terms-Adaptive feedback synchronization, complex networks, delayed nodes.

\section{INTRODUCTION}

$\mathbf{T}$ HE so-called complex network refers to a set of nodes connected by edges (graph) that has certain nontrivial topological features that are not found in simple networks [1]-[4]. Such nontrivial features involve a degree distribution with a heavy-tail, a hierarchical structure, a high clustering coefficient, a community structure at different scales, and assortativity or disassortativity among vertices [2], [5]-[8]. It is well known that complex networks exist in many natural and man-made systems, e.g., food webs, neural networks, cellular and metabolic networks, electrical power grids, computer networks, technological networks, the World Wide Web, coauthorship and citation networks, social networks, etc. [1], [2].

Time delay inevitably exists in natural and man-made networks [9]-[15]. In much of the literature, time delays in the cou-

Manuscript received May 25, 2007; revised September 17, 2007. This work was supported by National Natural Science Foundation of China under Grants 60574045, 70771084, 60221301 and 60772158, by National Basic Research (973) Program of China under Grant 2007CB310800 and 2007CB310805, by Important Direction Project of Knowledge Innovation Program of Chinese Academy of Sciences under Grant KJCX3-SYW-S01, and by Scientific Research Startup Special Foundation on Excellent PhD Thesis, and Presidential Award of Chinese Academy of Sciences. This paper was recommended by Associate Editor J. Suykens.

Q. Zhang and J. Lu are with the School of Mathematics and Statistics, Wuhan University, Wuhan 430072, China (e-mail: qunjiao99@163.com, jalu@whu.edu.cn).

J. Lü is with the Institute of Systems Science, Academy of Mathematics and Systems Science, Chinese Academy of Sciences, Beijing 100080, China, and also with the State Key Laboratory of Software Engineering, Wuhan University, Wuhan 430072, China (e-mail: jhlu@iss.ac.cn).

C. K. Tse is with the Department of Electronic and Information Engineering, Hong Kong Polytechnic University, Hong Kong, China (e-mail: encktse@ polyu. edu.hk).

Digital Object Identifier 10.1109/TCSII.2007.911813 plings (edges) are considered [9]-[11]; however, the time delays in the dynamical nodes [12]-[15], which are more complex, are still relatively unexplored. As a matter of fact, one can find numerous examples in the real world which are characterized by delayed differential equations having time delays in the dynamical nodes [12]-[15]. For example, the delayed logistic differential equation, which has time delay in the dynamical node, is a representative dynamical model of the electrochemical intercalations and physiological systems [15]. It is thus imperative to further investigate complex dynamical networks with delayed nodes. However, such complex networks are still relatively unexplored due to their complexity and the absence of an appropriate simplification procedure [9], [10]. Further, the lack of a general approach or tool to study such kind of complex networks has also obstructed the progress of development of their analysis [11]. Recently, we developed a method to deal with such kind of complex networks [16], and in this paper we further investigate the synchronization of a general complex dynamical network with delayed nodes.

Synchronization is now widely regarded as a kind of collective behavior which is exhibited in many natural systems [1], [16], [17]. In essence, synchronization is a form of self-organization. It has been demonstrated that many real-world problems have close relationships with network synchronization [1], [2], [8]. For example, theoretical and experimental results show that a mammalian brain not only displays in its storage of associative memories, but also modulates oscillatory neuronal synchronization by selective perceive attention [18].

Recently, synchronization of complex dynamical networks has been a focus in various fields of science and engineering. $\mathrm{Wu}[5]$ investigated the synchronization of random directed networks. Lü and Chen [8] studied the synchronization of time-varying complex dynamical networks. Li et al. [9], [11] explored the synchronization of complex dynamical networks with nonlinear inner-coupling functions and time delays. Zhou et al. [16] studied the adaptive synchronization of an uncertain complex dynamical network. Sorrentino et al. [17] investigated the controllability of complex networks with pinning controllers. However, the important issue of synchronization of complex dynamical networks with delayed nodes has only been lightly covered [9]-[15]. This paper will further investigate the adaptive feedback synchronization of complex dynamical networks with delayed nodes. In particular, we obtain several novel criteria for globally exponentially asymptotic synchronization. It should be pointed out that our hypotheses and the proposed adaptive controllers for network synchronization are very simple and easy to apply.

This paper is organized as follows. Section II introduces a general complex dynamical network with delayed nodes and several useful hypotheses. A set of novel adaptive feedback synchronization criteria are given in Section III. Section IV uses two 
representative examples to show the effectiveness of the proposed synchronization criteria. Some concluding remarks are given in Section $\mathrm{V}$.

\section{PROBLEM Formulation AND PRELIMINARIES}

We consider a general complex network consisting of $N$ delayed dynamical nodes [7], [8]. Each node of the network is an $n$-dimensional nonautonomous dynamical system with time delay, which is described by

$$
\dot{x}_{i}(t)=f\left(x_{i}(t), x_{i}(t-\tau)\right)+\sum_{j=1}^{N} c_{i j} A x_{j}(t)+U_{i}
$$

where $i=1,2, \ldots, N, x_{i}(t)=\left(x_{i 1}(t), x_{i 2}(t), \cdots, x_{i n}(t)\right)^{T}$ $\in \mathbf{R}^{n}$ are the state variables of node $i, \tau>0$ is the constant time delay, and $c_{i j}$ are the directed couplings from nodes $i$ to $j$. The matrix $A=\left(a_{i j}\right)_{n \times n} \in \mathbf{R}^{n \times n}$ is the inner connecting matrix of each node and the matrix $C=\left(c_{i j}\right)_{N \times N} \in \mathbf{R}^{N \times N}$ is the diffusively coupled matrix of the network. That is,

$$
c_{i i}=-\sum_{\substack{j=1 \\ \supset \neq i}}^{N} c_{i j}
$$

where $c_{i j} \geq 0(i \neq j)$ represent the coupling strengths from nodes $i$ to $j$. Moreover, $U_{i} \in \mathbf{R}^{n}$ are the controllers designed for the network.

Remark 1: In the network (1), the outer-coupling matrix $C$ is not necessarily to be symmetric and the elements $c_{i j}$ are not assumed to be only 0 or 1 . Moreover, there is no any constraint on the inner-coupling matrix $A$.

Before starting the main results, some necessary definitions and assumptions are given in the following.

Assumption $1(A 1)$ : For the delayed differential equation

$$
\dot{x}(t)=f(x(t), x(t-\tau))
$$

where $x(t) \in \mathbf{R}^{n}, f: \mathbf{R}^{n} \times \mathbf{R}^{n} \rightarrow \mathbf{R}^{n}$ is a continuous function, there exists a unique continuous solution for any initial condition $\left(t_{0}, x^{0}\right)$, where $x^{0}$ is an $n$-dimensional vector.

Assumption 2(A2): For the vector function $f(x(t), x(t-\tau)$ ), suppose that the uniform Lipschitz condition holds, i.e., for any $x_{i}(t)=\left(x_{i 1}(t), x_{i 2}(t), \cdots, x_{i n}(t)\right)^{T}$ and $s(t)=\left(s_{1}(t), s_{2}(t), \cdots, s_{n}(t)\right)^{T}$. Then, there exists a positive constant $L>0$, such that

$$
\begin{aligned}
& \left\|f\left(x_{i}(t), x_{i}(t-\tau)\right)-f(s(t), s(t-\tau))\right\| \\
& \quad \leq L\left[\left\|x_{i}(t)-s(t)\right\|+\left\|x_{i}(t-\tau)-s(t-\tau)\right\|\right],
\end{aligned}
$$

where $i=1,2, \ldots N$.

Hereafter, $\|x(t)\|=\sqrt{x_{1}^{2}(t)+x_{2}^{2}(t)+\cdots+x_{n}^{2}(t)}$ and the spectral norm of matrix $A$ is defined by $\|A\|=\sqrt{\lambda_{\max }\left(A^{T} A\right)}$. Assume that $s(t)$ is a solution of the node system (3) satisfying

$$
\dot{s}(t)=f(s(t), s(t-\tau))
$$

where $s(t)$ may be an equilibrium point, a periodic orbit, an aperiodic orbit, or a chaotic orbit in the phase space.

Definition 1: [4], [7], [8]. Let $x_{i}\left(t, \tau ; t_{0}, X_{0}\right)(1 \leq i \leq N)$ be a solution of the controlled network (1), where $\overline{X_{0}}=$ $\left(x_{1}^{0}, x_{2}^{0}, \cdots, x_{N}^{0}\right) \in \mathbf{R}^{n \times N}$. Assume that $f: \Omega \times \Omega \rightarrow \mathbf{R}^{n}$ and
$U_{i}: \Omega \times \cdots \times \Omega \rightarrow \mathbf{R}^{n}(1 \leq i \leq N)$ are continuous, $\Omega \subseteq \mathbf{R}^{n}$. If there is a nonempty subset $\Gamma \subseteq \Omega$, with $x_{i}^{0} \in \Gamma(1 \leq i \leq N)$, such that $x_{i}\left(t, \tau ; t_{0}, X_{0}\right) \in \Omega$ for all $t \geq t_{0}, 1 \leq i \leq N$, and

$$
\lim _{t \rightarrow \infty}\left\|x_{i}\left(t, \tau ; t_{0}, X_{0}\right)-s\left(t, \tau ; t_{0}, x_{0}\right)\right\|=0
$$

where $1 \leq i \leq N$ and $x_{0} \in \Omega$, then the controlled network (1) is said to achieve asymptotical network synchronization and $\Gamma \times \cdots \times \Gamma$ is called the region of synchrony for the dynamical network (1).

Define error vectors as

$$
e_{i}(t)=x_{i}(t)-s(t)
$$

where $1 \leq i \leq N$. According to the controlled network (1) and notice that $\sum_{j=1}^{N} c_{i j}=0$, the error system is then described by

$$
\begin{aligned}
\dot{e}_{i}(t)= & \dot{x}_{i}(t)-\dot{s}(t) \\
= & f\left(x_{i}(t), x_{i}(t-\tau)\right)+\sum_{j=1}^{N} c_{i j} A x_{j}(t)+U_{i} \\
& -f(s(t), s(t-\tau))-\sum_{j=1}^{N} c_{i j} A s(t) \\
= & f\left(x_{i}(t), x_{i}(t-\tau)\right)-f(s(t), s(t-\tau)) \\
& +\sum_{j=1}^{N} c_{i j} A e_{j}(t)+U_{i}
\end{aligned}
$$

where $1 \leq i \leq N$.

Definition 2: The network (1) is said to be globally exponentially asymptotical synchronous if there exist constants $M>0$ and $\alpha>0$, such that for any initial condition,

$$
\left\|e_{i}(t)\right\| \leq M \exp (-\alpha t)
$$

where $i=1,2, \cdots, N$ and $e_{i}(t)=x_{i}(t)-s(t)$.

In the following, the main goal is to design appropriate adaptive controllers $U_{i}$ and the corresponding updating laws which make the network (1) globally exponentially asymptotically synchronous.

\section{ADAPTIVE FEEDBACK SYNCHRONIZATION OF A GENERAL COMPLEX DYNAMICAL NETWORK WITH DELAYED NODES}

In this section, we will introduce several useful adaptive feedback synchronization criteria for the complex dynamical network (1) with delayed nodes.

Theorem 1: Suppose that A1 and $\mathbf{A} \mathbf{2}$ hold. Let the controllers be $U_{i}=-d_{i} e_{i}(i=1,2, \ldots, N)$ with the following updating laws:

$$
\dot{d}_{i}=\delta_{i}\left\|e_{i}(t)\right\|^{2} \exp (\mu t)
$$

where $\mu$ and $\delta_{i}$ are positive constants. Then, the controlled network (1) is globally exponentially asymptotically synchronous. Moreover,

$$
\left\|e_{i}(t)\right\| \leq M \exp \left(-\frac{\mu}{2} t\right) \text { with } M>0 .
$$


Proof: Construct the Lyapunov function candidate as follows:

$$
\begin{aligned}
V(t)=\frac{1}{2} & {\left[\sum _ { i = 1 } ^ { N } \left(e_{i}^{T}(t) e_{i}(t) \exp (\mu t)+L \int_{t-\tau}^{t} e_{i}^{T}(\theta) e_{i}(\theta)\right.\right.} \\
& \times \exp (\mu(\theta+\tau)) \mathrm{d} \theta)]+\frac{1}{2} \sum_{i=1}^{N} \frac{\left(d_{i}-d_{i}^{\star}\right)^{2}}{\delta_{i}} .
\end{aligned}
$$

By using the inequality $x^{T} y \leq\left|x^{T} y\right| \leq\|x \mid\| y \|$, along with (8) and (10), we have

$$
\begin{aligned}
& \dot{V}(t)=\sum_{i=1}^{N}\left[e_{i}^{T}(t) \dot{e}_{i}(t) \exp (\mu t)+\frac{\mu}{2} e_{i}^{T}(t) e_{i}(t) \exp (\mu t)\right. \\
& +\frac{L}{2}\left(e_{i}^{T}(t) e_{i}(t) \exp (\mu \tau)-e_{i}^{T}(t-\tau) e_{i}(t-\tau)\right) \\
& * \exp (\mu t)]+\sum_{i=1}^{N} \frac{1}{\delta_{i}}\left(d_{i}-d_{i}^{\star}\right) \dot{d}_{i} \\
& =\sum_{i=1}^{N}\left\{\left[e_{i}^{T}(t) \dot{e}_{i}(t)+\frac{\mu}{2}\left\|e_{i}(t)\right\|^{2}+\frac{L}{2}\right.\right. \\
& \left.\times\left(\left\|e_{i}(t)\right\|^{2} \exp (\mu \tau)-\left\|e_{i}(t-\tau)\right\|^{2}\right)\right] \\
& \left.+\left(d_{i}-d_{i}^{\star}\right)\left\|e_{i}(t)\right\|^{2}\right\} \exp (\mu t) \\
& =\sum_{i=1}^{N}\left\{e _ { i } ^ { T } ( t ) \left[f\left(x_{i}(t), x_{i}(t-\tau)\right)-f(s(t), s(t-\tau))\right.\right. \\
& \left.+\sum_{j=1}^{N} c_{i j} A e_{j}(t)-d_{i} e_{i}\right]+\frac{\mu}{2}\left\|e_{i}(t)\right\|^{2} \\
& +\frac{L}{2}\left(\left\|e_{i}(t)\right\|^{2} \exp (\mu \tau)-\left\|e_{i}(t-\tau)\right\|^{2}\right) \\
& \left.+\left(d_{i}-d_{i}^{\star}\right)\left\|e_{i}(t)\right\|^{2}\right\} \exp (\mu t) \\
& \leq \sum_{i=1}^{N}\left\{\| e _ { i } ( t ) \| \left[\left\|f\left(x_{i}(t), x_{i}(t-\tau)\right)-f(s(t), s(t-\tau))\right\|\right.\right. \\
& +\sum_{j=1}^{N}\left|c_{i j}\|\mid A\|\left\|e_{j}(t)\right\|\right]+\frac{\mu}{2}\left\|e_{i}(t)\right\|^{2} \\
& +\frac{L}{2}\left(\left\|e_{i}(t)\right\|^{2} \exp (\mu \tau)-\left\|e_{i}(t-\tau)\right\|^{2}\right) \\
& \left.-d_{i}^{\star}\left\|e_{i}(t)\right\|^{2}\right\} \exp (\mu t) \\
& \leq \sum_{i=1}^{N}\left\{\| e _ { i } ( t ) \| \left[L\left(\left\|e_{i}(t)\right\|+\left\|e_{i}(t-\tau)\right\|\right)\right.\right. \\
& \left.+\sum_{j=1}^{N}\left|c_{i j}\right|\|A\|\left\|e_{j}(t)\right\|\right]+\frac{\mu}{2}\left\|e_{i}(t)\right\|^{2} \\
& +\frac{L}{2}\left(\left\|e_{i}(t)\right\|^{2} \exp (\mu \tau)-\left\|e_{i}(t-\tau)\right\|^{2}\right)
\end{aligned}
$$

$$
\left.-d_{i}^{\star}\left\|e_{i}(t)\right\|^{2}\right\} \exp (\mu t) .
$$

Denote $c_{i}=\max _{1 \leq k \leq N}\left|c_{i k}\right|(i=1,2, \ldots, N)$, and take account into the fact $2 a b \leq a^{2}+b^{2}$, we further have

$$
\begin{aligned}
& \dot{V}(t) \leq \sum_{i=1}^{N}\left[L\left\|e_{i}(t)\right\|^{2}+\frac{L}{2}\left(\left\|e_{i}(t)\right\|^{2}+\left\|e_{i}(t-\tau)\right\|^{2}\right)\right. \\
& +\|A\| c_{i} \sum_{j=1}^{N}\left\|e_{i}(t)\right\|\left\|e_{j}(t)\right\|-\frac{L}{2}\left\|e_{i}(t-\tau)\right\|^{2} \\
& \left.+\left(\frac{\mu}{2}+\frac{L}{2} \exp (\mu \tau)-d_{i}^{\star}\right)\left\|e_{i}(t)\right\|^{2}\right] \exp (\mu t) \\
& \leq \sum_{i=1}^{N}\left[\|A\| c_{i} \sum_{j=1}^{N}\left(\frac{\left\|e_{i}(t)\right\|^{2}+\left\|e_{j}(t)\right\|^{2}}{2}\right)\right. \\
& \left.+\left(\frac{3}{2} L+\frac{\mu}{2}+\frac{L}{2} \exp (\mu \tau)-d_{i}^{\star}\right)\left\|e_{i}(t)\right\|^{2}\right] \\
& \times \exp (\mu t) \\
& =\sum_{i=1}^{N}\left\{\left[\frac{3}{2} L+\frac{\mu}{2}-d_{i}^{\star}+\frac{L}{2} \exp (\mu \tau)+\frac{N\|A\| c_{i}}{2}\right.\right. \\
& \left.\left.+\frac{\|A\|}{2} \sum_{j=1}^{N} c_{j}\right] \cdot\left\|e_{i}(t)\right\|^{2} \exp (\mu t)\right\} .
\end{aligned}
$$

Select suitable constants $d_{i}^{\star}(i=1,2, \ldots N)$ satisfying

$$
\frac{3}{2} L+\frac{\mu}{2}-d_{i}^{\star}+\frac{L}{2} \exp (\mu \tau)+\frac{N\|A\| c_{i}}{2}+\frac{\|A\|}{2} \sum_{j=1}^{N} c_{j}<0 .
$$

Then, we have $\dot{V}(t) \leq 0$. It follows that $V(t) \leq V(0)$ for any $t \geq 0$.

Using the Lyapunov function (12), we have

$$
\begin{aligned}
\frac{1}{2}\left\|e_{i}(t)\right\|^{2} \exp (\mu t) & =\frac{1}{2} e_{i}^{T}(t) e_{i}(t) \exp (\mu t) \\
& \leq V(t) \leq V(0) .
\end{aligned}
$$

Therefore, we obtain

$$
\left\|e_{i}(t)\right\| \leq M \exp \left(-\frac{\mu}{2} t\right) \quad \text { with } \quad M=\sqrt{2 V(0)}>0 .
$$

Thus, $\lim _{t \rightarrow \infty}\left\|e_{i}(t)\right\|=0$. That is, the controlled network (1) is globally exponentially asymptotically synchronous. Q.E.D.

Theorem 2: Suppose that $\mathbf{A 1}$ and $\mathbf{A 2}$ hold. Let the controllers be $U_{i}=-d_{i} e_{i}(i=1,2, \ldots N)$ with the following updating laws:

$$
\dot{d}_{i}=\delta_{i}\left\|e_{i}(t)\right\| \exp (\mu t)
$$

where $\mu$ and $\delta_{i}$ are positive constants, then the controlled network (1) is globally exponentially asymptotically synchronous. Furthermore,

$$
\left\|e_{i}(t)\right\| \leq M \exp (-\mu t) \quad \text { with } \quad M>0 .
$$


Proof: Construct the Lyapunov function candidate as follows:

$$
\begin{aligned}
& V(t)=\sum_{i=1}^{N}\left[\left\|e_{i}(t)\right\| \exp (\mu t)\right. \\
& \left.+L \int_{t-\tau}^{t}\left\|e_{i}(\theta)\right\| \exp (\mu(\theta+\tau)) \mathrm{d} \theta\right]+\frac{1}{2} \sum_{i=1}^{N} \frac{\left(d_{i}-d_{i}^{\star}\right)^{2}}{\delta_{i}} .
\end{aligned}
$$

Since $\left\|e_{i}(t)\right\|^{\prime}=\left(e_{i}^{T}(t) \dot{e}_{i}(t) /\left\|e_{i}(t)\right\|\right)$, the Dini derivative of $V(t)$ with respect to time $t$ along the trajectories of (8) is then given by

$$
\begin{aligned}
\dot{V}(t)= & \sum_{i=1}^{N}\left[\frac{e_{i}^{T}(t) \dot{e}_{i}(t)}{\left\|e_{i}(t)\right\|} \exp (\mu t)+\mu\left\|e_{i}(t)\right\| \exp (\mu t)\right. \\
& \left.+L\left(\left\|e_{i}(t)\right\| \exp (\mu \tau)-\left\|e_{i}(t-\tau)\right\|\right) \exp (\mu t)\right] \\
+ & \sum_{i=1}^{N} \frac{1}{\delta i}\left(d_{i}-d_{i}^{\star}\right) \dot{d}_{i} \\
\leq & \sum_{i=1}^{N}\left[L\left(\left\|e_{i}(t)\right\|+\left\|e_{i}(t-\tau)\right\|\right)+\|A\| c_{i} \sum_{j=1}^{N}\left\|e_{j}(t)\right\|\right. \\
& \left.\quad-L\left\|e_{i}(t-\tau)\right\|+\left(\mu+L \exp (\mu \tau)-d_{i}^{\star}\right)\left\|e_{i}(t)\right\|\right] \\
= & \sum_{i=1}^{N}\left[L+\mu-d_{i}^{\star}+L \exp (\mu \tau)+\|A\| \sum_{j=1}^{N} c_{j}\right] \\
& \cdot\left\|e_{i}(t)\right\| \exp (\mu t) .
\end{aligned}
$$

Now, choose suitable constants $d_{i}^{\star}(i=1,2, \ldots N)$ satisfying

$$
L+\mu-d_{i}^{\star}+L \exp (\mu \tau)+\|A\| \sum_{j=1}^{N} c_{j}<0 .
$$

Therefore, $\dot{V}(t) \leq 0$. Similar to the proof of Theorem 1, we have

$$
\left\|e_{i}(t)\right\| \leq M \exp (-\mu t) \quad \text { with } \quad M=\sqrt{2 V(0)}>0 .
$$

Thus, $\lim _{t \rightarrow \infty}\left\|e_{i}(t)\right\|=0$. That is, the controlled network (1) is globally exponentially asymptotically synchronous. Q.E.D.

\section{EXAMPLES}

In the foregoing section, Theorems 1 and 2 essentially provide the criteria for global exponential asymptotic synchronization. In this section, we use two representative examples to illustrate how these theorems can be applied to achieve synchronization in complex networks with delayed nodes.

Example 1: We first consider a simple network with 50 nodes and time delay $\tau=1$. The delayed dynamical equation of each node is described by

$$
\left\{\begin{array}{l}
\dot{x}_{i 1}(t)=a\left(x_{i 2}(t)-x_{i 1}(t)\right) \\
\dot{x}_{i 2}(t)=c x_{i 1}(t)-x_{i 1}(t) x_{i 3}(t-1)-x_{i 2}(t) \\
\dot{x}_{i 3}(t)=x_{i 1}(t) x_{i 2}(t-1)-b x_{i 3}(t-1)
\end{array}\right.
$$

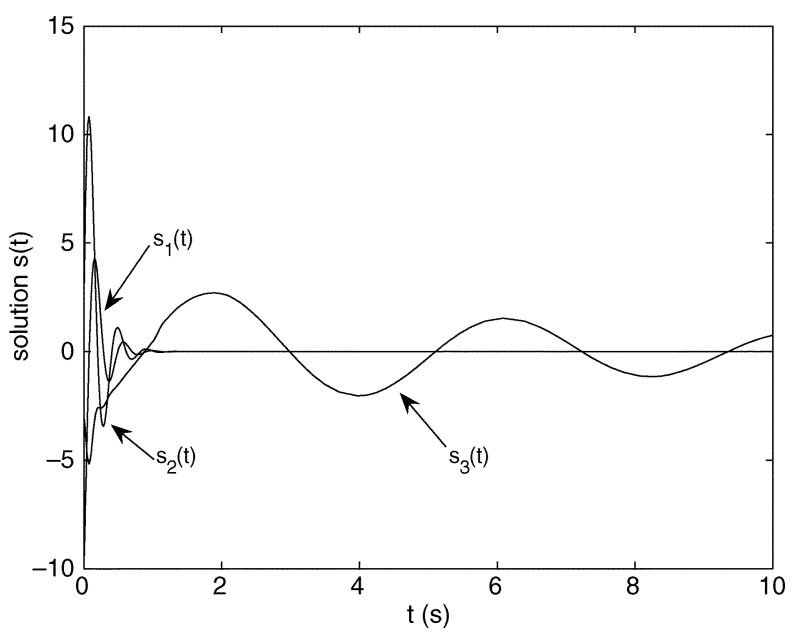

Fig. 1. Solution $s(t)$ of the delayed differential equations (24) with initial values vector $[-102-3]^{T}$.
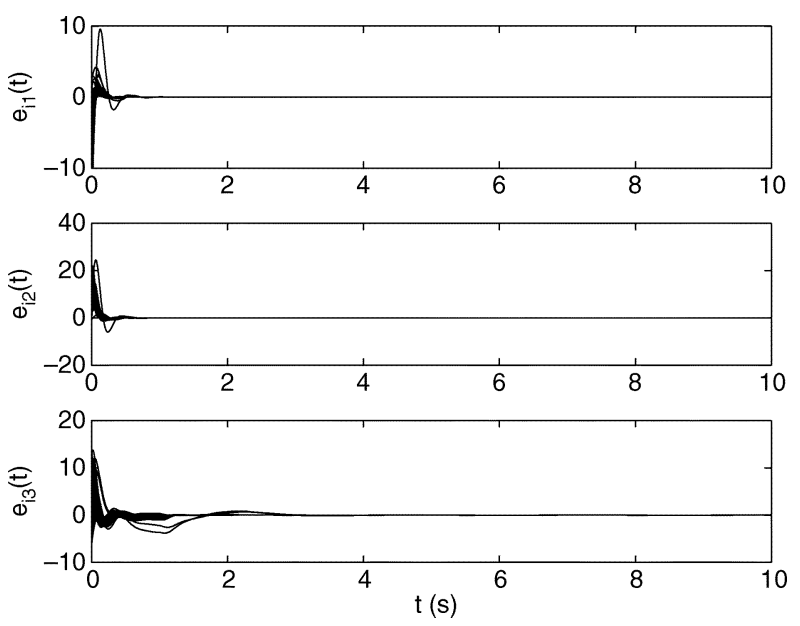

Fig. 2. Synchronization errors $e_{i 1}, e_{i 2}, e_{i 3}(i=1,2, \ldots, 50)$ under updating law (10).
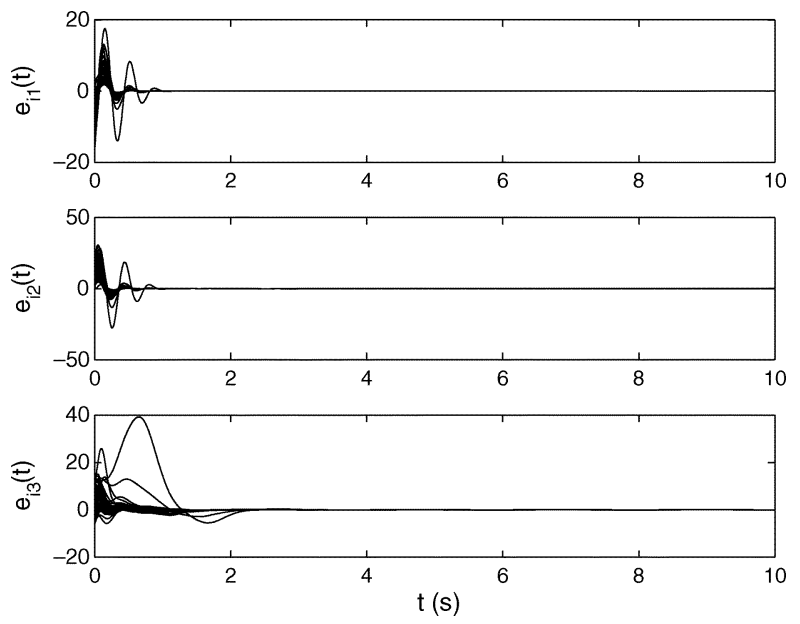

Fig. 3. Synchronization errors $e_{i 1}, e_{i 2}, e_{i 3}(i=1,2, \ldots, 50)$ under updating law (18).

where $i=1,2, \ldots 50, a=10, b=1.3$ and $c=-28$.

For the initial vector value $x^{0}=(-10,2,-3)^{T}$, the solution of system (24) is denoted by $s(t)=\left(s_{1}(t), s_{2}(t), s_{3}(t)\right)^{T}$, which is shown in Fig. 1. 


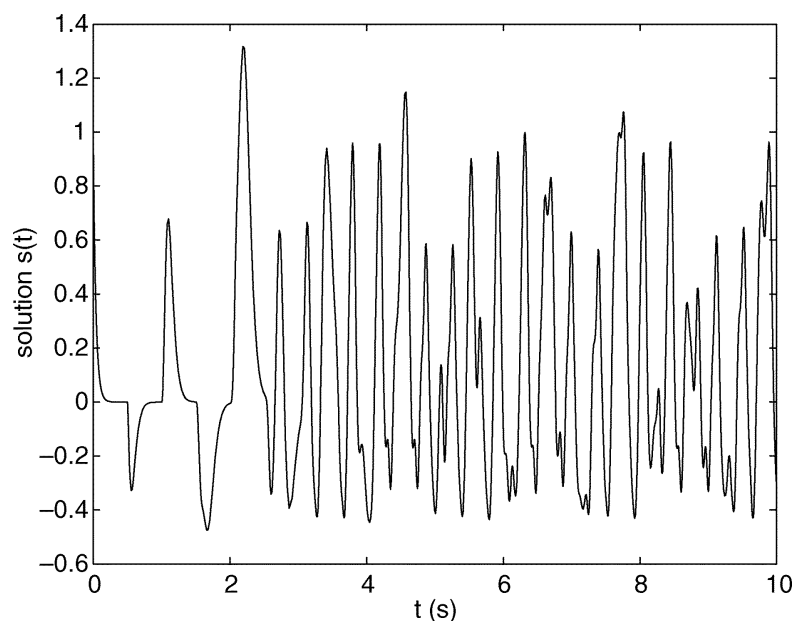

Fig. 4. Solution $s(t)$ of the delayed logistic differential equation (25) with initial value $x^{0}=1$.
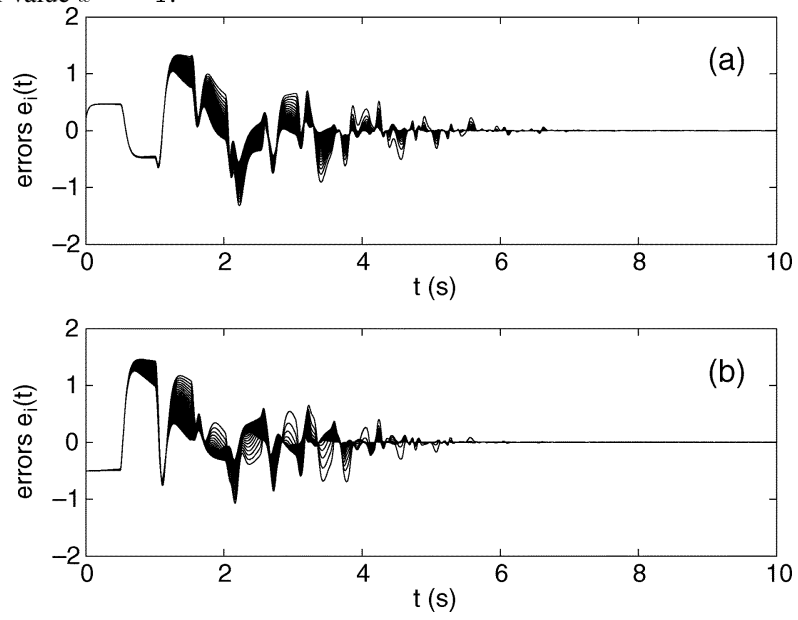

Fig. 5. Synchronization errors $e_{i}(t)(i=1,2, \ldots, 50)$ of the delayed logistic network with adaptive feedback controllers under (a) updating law (10); and (b) updating law (18).

Here, we assume that these nodes are globally connected with weighted edges. For convenience, select $c_{i j}=0.1(i \neq j)$. The coupling configuration matrix is thus given by

$$
C=\left(\begin{array}{ccccc}
-4.9 & 0.1 & 0.1 & \ldots & 0.1 \\
0.1 & -4.9 & 0.1 & \ldots & 0.1 \\
\vdots & \vdots & \vdots & \vdots & \vdots \\
0.1 & 0.1 & \ldots & -4.9 & 0.1 \\
0.1 & 0.1 & \ldots & 0.1 & -4.9
\end{array}\right)
$$

Assume that the inner matrix $A=I_{3}$ and the Lipchitz constant $L=46.14$. Obviously, assumptions $\mathbf{A 1}$ and $\mathbf{A 2}$ hold.

If $d_{i}^{*}(i=1,2, \ldots, 50)$ are large enough, according to Theorems 1 and 2, then the inequalities (15) and (22) hold. Let $\delta_{i}=0.1 * i(i=1,2, \ldots, 50)$. Fig. 2 and 3 show the synchronization errors of $e_{i 1}(t), e_{i 2}(t), e_{i 3}(t)$ under the updating laws (10) and (18), respectively. Clearly, all synchronization errors are rapidly converging to zero.

Example 2: Next we consider a more complex node system that exhibits chaotic behavior. Specifically, we examine the logistic delay differential equation that characterizes the nonlinear dynamics of the electrochemical intercalations and physiological systems [15]. The logistic delay differential equation of each node is given by [15]

$$
\dot{x}_{i}(t)=-\alpha x_{i}(t)+r x_{i}(t-\tau)\left(1-x_{i}(t-\tau)\right)
$$

where $i=1,2, \ldots 50$. When $\alpha=26, \tau=0.5$ and $r=-53$, system (25) is chaotic, as shown in Fig. 4, where the initial value $x^{0}=1$.

The coupling configuration matrix $C$ and the constants $\delta_{i}$ $(i=1,2, \ldots, 50)$ are as given in the earlier example. The synchronization errors $e_{i}(t)(i=1,2, \ldots, 50)$ of the delayed logistic network under the updating laws (10) and (18) are shown in Fig. 5(a) and (b), respectively. All synchronization errors rapidly converge to zero.

\section{CONCLUSION}

A general complex dynamical network with delayed nodes has been studied in this paper. Such a network represents a realistic form of networks which has not been thoroughly addressed previously. Specifically, by constructing appropriate Lyapunov functions, several novel adaptive feedback synchronization criteria are derived. These criteria are very useful for understanding the mechanism of synchronization in complex networks with time delayed nodes. Moreover, the hypotheses and the resulting adaptive controllers for achieving network synchronization are expressed in simple forms that can be readily applied in practical situations. Finally, numerical simulations have been presented to demonstrate the effectiveness of the proposed synchronization criteria.

\section{REFERENCES}

[1] S. H. Strogatz, "Exploring complex networks," Nature, vol. 410, no. 6825, pp. 268-276, Mar. 2001

[2] R. Albert and A.-L. Barabási, "Statistical mechanics of complex networks," Rev. Mod. Phys., vol. 74, no. 1, pp. 47-97, Jan. 2002

[3] H. Hong, M. Y. Choi, and B. J. Kim, "Synchronization on small-world networks," Phys. Rev. E, vol. 65, no. 5, pp. 26-139, Jan. 2002.

[4] J. Lü, X. H. Yu, G. Chen, and D. Z. Cheng, "Characterizing the synchronizability of small-world dynamical networks," IEEE Trans. Circuits Syst. I, Reg. Papers, vol. 51, no. 4, pp. 787-796, Apr. 2004.

[5] C. W. Wu, "Synchronization and convergence of linear dynamics in random directed networks," IEEE Trans. Autom. Control, vol. 51, no. 7, pp. 1207-1210, Jul. 2006.

[6] Z. Li and G. Chen, "Global synchronization and asymptotic stability of complex dynamical networks," IEEE Trans. Circuits Syst. II, Exp. Briefs, vol. 53, no. 1, pp. 28-33, Jan. 2006.

[7] J. Lü, X. Yu, and G. Chen, "Chaos synchronization of general complex dynamical networks," Phys. A, vol. 334, no. 1-2, pp. 281-302, Mar. 2004.

[8] J. Lü and G. Chen, "A time-varying complex dynamical network model and its controlled synchronization criteria," IEEE Trans. Autom. Control, vol. 50, no. 6, pp. 841-846, Jun. 2005.

[9] C. Li, H. Xu, X. Liao, and J. Yu, "Synchronization in small-world oscillator networks with coupling delays," Phys. A, vol. 335, no. 3-4, pp 359-364, Apl. 2004

[10] J. Zhou, Z. Liu, and G. Chen, "Dynamics of delayed nerual networks," Neur. Networks, vol. 17, no. 1, pp. 87-101, Jan. 2004.

[11] C. Li, S. Li, X. Liao, and J. Yu, "Synchronization in coupled map lattices with small-world delayed interactions," Phys. A, vol. 335, no. 3-4, pp. 365-370, Apr. 2004

[12] B. Mensour and A. Longtin, "Synchronization of delay-differential equations with application to private communication," Phys. Lett. A vol. 244, no. 1-3, pp. 59-70, Jul. 1998.

[13] K. Pyragas, "Synchronization of coupled time-delay systems: Analytical estimations," Phys. Rev. E, vol. 58, no. 3, pp. 3067-3071, Sep. 1998.

[14] C. Li, X. Liao, and K. Wong, "Chaotic lag synchronization of coupled time-delayed systems and its applications in secure communication," Phys. D, vol. 194, no. 3-4, pp. 187-202, Jul. 2004.

[15] M. Jiang, Y. Shen, J. Jian, and X. Liao, "Stability, bifurcation and a new chaos in the logistic differential equation with delay," Phys. Lett. A, vol. 350, no. 3-4, pp. 221-227, Feb. 2006.

[16] J. Zhou, J. Lu, and J. Lü, "Adaptive synchronization of an uncertain complex dynamical network," IEEE Trans. Autom. Control, vol. 51, no. 4, pp. 652-656, Apr. 2006

[17] F. Sorrentino, M. di Bernardo, F. Garofalo, and G. Chen, "Controllability of complex networks via pinning," Phys. Rev. E, vol. 75, no. 4, p. 046103, 2007.

[18] M. V. L. Bennett and R. S. Zukin, "Electrical coupling and neuronal synchronization in the mammalian brain," Neuron, vol. 41, no. 4, pp. 495-511, Feb. 2004 